\title{
Potential problems of inadequate air removal and presence of non-condensable gasses in a steam sterilization process: A brief discussion
}

\author{
Tapan Kumar Das BSc, DHM, MBA ${ }^{1}$, Samir Kumar Laha BSc (Math Hons)² and Debabrata Basu MSc (Evns), DHM³ (1) \\ ${ }^{1}$ Central Sterile Services Department, Apollo Gleneagles Hospital, Kolkata, West Bengal, India, ${ }^{2}$ Central Sterile Services Department, Peerless Hospital, Kolkata, \\ West Bengal, India and ${ }^{3}$ Central Sterile Services Department, Tata Medical Center, Kolkata, West Bengal, India
}

To the Editor-Medical devices that are reprocessed in a sterile processing department must be cleaned, disinfected, and sterilized to prevent infection before apply to the patient. The preferred and most commonly used sterilization method is steam sterilization, in which saturated steam (moist heat in the form of saturated steam under pressure) is used as a sterilizing agent. ${ }^{1}$ The steam sterilization process has several advantages: it is inexpensive, nontoxic, fast, accurate, and widely recognized. The basic principle of the steam sterilization process is to remove air and noncondensable gases (NCGs) from the sterilization chamber by gravitational system or by separate vacuum pump so that steam gets easy access for sterilization. However, when air removal is insufficient, the sterilization process is suboptimal, even if all other process parameters, such as steam pressure and temperature, are achieved. Therefore, the quality of the vacuum pump in relation to the chamber volume is very important for proper air removal and steam penetration purposes. In addition, door seals, gaskets, and other valves should be tested frequently to ensure that there is no leakage where air and NCGs (ie, carbon dioxide and hydrogen) can enter during the sterilization period. ${ }^{2}$

The presence of air and other NCGs inside the sterilization chamber is one of the biggest threats to the sterilization process. NCGs prevent steam from reaching the medical device, inhibiting thermal coagulation of spores during sterilization. Therefore, air pockets form (bubbles of NCGs) that isolate the goods to be sterilized and block further condensation of the steam before sterilization. In addition, formation of NCGs inside the sterilizer chamber (NCGs should be $\leq 0.15 \%$ ) can slow down the heating process or the sterilization cycle may abort due to insufficient vacuum if the gases are not properly removed.

No reliable equipment monitoring device is available to monitor insufficient air removal inside the sterilizer chamber. However, operators can check the temperature and pressure against time via thermoelectrical measurement to interpret how much vacuum are achieved according to the sterilizer manufacturer. The measurement is based on simultaneous measurement of the temperature in the center of the pack and outside it. Some additional preventive actions can be taken to reduce the problem: (1) using a good quality vacuum pump because adequate air removal by good quality vacuum pump reduces the residual air in the

\footnotetext{
Author for correspondence: Debabrata Basu, E-mail: debabrata.basu@tmckolkata.com Cite this article: Das TK, Laha SK, and Basu D. (2020). Potential problems of inadequate air removal and presence of non-condensable gasses in a steam sterilization process: A brief discussion. Infection Control \& Hospital Epidemiology, 41: 739-740, https://doi.org/10.1017/ice.2020.88

chamber; (2) increasing the number of pulse-vacuum phase, which is determined by capacity of vacuum pump with chamber volume or density of the sterilizer; (3) performing timely maintenance of door gaskets, seals, and valves because door gaskets can be leaked by a high level of contaminants or uneven surface of sterilizer door and valves can be damaged by continuous use of sterilizer; and (4) using cold water (for deep vacuum) with a deionization system because hydrogen carbonate containing water can produce $\mathrm{CO}_{2}$ and carbonate if the ion exchanger fails during steam preparation. ${ }^{3}$

In 1963, the Bowie-Dick or air removal test for vacuum-assist sterilizers was developed to detect air and NCGs present in a sterilization cycle. To confirm their absence, an air removal cycle is run first in an empty chamber with exposure time of 3.5 minutes at $134^{\circ} \mathrm{C} .{ }^{4}$ If the Bowie-Dick test is passed satisfactorily, then the following load cycle run with a biological indicator to monitor the lethality of a given sterilization process for biologically proven sterility assurance. Every test device should be placed inside the sterilizer chamber in the most challenging area, (eg, in front of the door or drain strainer) for a worst-case scenario. If the air is correctly removed, then steam can penetrate satisfactorily and the color of the chemical indicator changes significantly and uniformly.

\section{Discussion}

The Bowie-Dick test is an equipment-specific test that assesses how well the autoclave vacuum pump is working according to EN 285, the European large steam sterilizer standard. It is the only indicator in which air and NCGs are detected. The test pack is composed of 3 important elements: (1) a porous barrier layer with standardized porosity, (2) an indicator layer composed of a type- 2 chemical indicator with thermochromic ink to detect steam quality, and (3) 2 transparent gas impervious layers composed of 2 polyester films with $0.01-0.03 \mathrm{~mm}$ thickness between which the indicator is placed. ${ }^{5}$ According to the weight of $[4 \mathrm{~kg}$, according to the American medical instrumentation standard] (AAMI/ ANSI ST79), or $7 \mathrm{~kg}$, according to the European (EN) and international (ISO) medical instrumentation standards, this test assesses the vacuum pump capacity, that how much air can be removed from the chamber and the individual package so that steam can penetrate well for proper sterilization. .,7 $^{6}$

Continuous quality monitoring of sterilization processes is of paramount importance in supplying sterile materials to patients. However a single Bowie-Dick test cannot give guarantee all over the day if the sterilizer is completely stopped for more than 3-4 hours at a time because NCGs (due to poor water quality) may accumulate inside the steam pipeline if water flow is stopped or stagnant. Moreover, even when the Bowie-Dick test has been successful, the process parameters can change with each subsequent 
Table 1. Advantages of Type-2 Chemical Indicator Used in Every Autoclave Cycle

\begin{tabular}{ll}
\hline Purpose & \multicolumn{1}{c}{ Advantages } \\
\hline $\begin{array}{l}\text { Sufficient temperature and steam } \\
\text { penetration in every cycle }\end{array}$ & $\begin{array}{l}\text { Avoids any uncertainty of air and } \\
\text { NCGs gases in the chamber }\end{array}$ \\
\hline $\begin{array}{l}\text { For porous and hollow items } \\
\text { sterilization }\end{array}$ & $\begin{array}{l}\text { Maximum air entrapment so that } \\
\text { the type-2 chemical indicator can } \\
\text { confirm the total elimination of air } \\
\text { and NCGs from the item }\end{array}$ \\
\hline $\begin{array}{l}\text { A secondary option after physical } \\
\text { monitoring }\end{array}$ & $\begin{array}{l}\text { Gives more accuracy by both } \\
\text { physical and chemical monitoring } \\
\text { and cross checks it }\end{array}$ \\
\hline $\begin{array}{l}\text { Condition of type-5 or yype-6 } \\
\text { chemical indicator inside the pack }\end{array}$ & $\begin{array}{l}\text { To avoid sterility check in every } \\
\text { package, type-2 chemical indicator } \\
\text { in a worst-case load can authorize } \\
\text { the entire load. }\end{array}$ \\
\hline $\begin{array}{l}\text { Incur minimal cost in every load } \\
\text { (Type-2 chemical indicator cost is } \\
\text { divided by number of items } \\
\text { sterilized in a load) }\end{array}$ & $\begin{array}{l}\text { Gives maximum output by } \\
\text { assuring complete dismissal of air } \\
\text { and NCGs for optimum sterility } \\
\text { assurance }\end{array}$ \\
\hline
\end{tabular}

Note. NCG, noncondensable gas.

cycle. Every delivery of steam to the sterilizer can have different characteristics that change in every cycle.

To ensure the air and NCG removal process and to control cost, we strongly recommend the use of a type- 2 chemical indicator in every cycle (Table 1 ) by a reusable test device according to the European standard for nonbiological systems for use in sterilizers part 5, specification for indicator systems and process challenge devices for use in performance testing for small sterilizers type B and type S (EN 867-5). This process can confirm that the sterilizer chamber and packages are free from air and NCGs to assure optimum sterility according to the European standard requirements for medical devices to be designated sterile (EN 556). ${ }^{8}$

\section{Acknowledgments. None.}

Financial support. No financial support was provided relevant to this article.

Conflicts of interest. All authors report no conflicts of interest relevant to this article.

\section{References}

1. Nagpal AK, Shriniwas AK. Principles of steam sterilization. Health Popul Perspect Issues 1978;1:40-50.

2. Winter S, Smith A, Lappin D, McDonagh G, Kirk B. Failure of nonvacuum steam sterilization processes for dental handpieces. J Hosp Infect 2017;97: 343-347.

3. van Wezel RAC, van Gastel A, de Ranitz A, van Doornmalen Gomez Hoyos JPCM. Following trends in steam sterilizer performance by quantitative monitoring of non-condensable gases. J Hosp Infect 2017;97:357-362.

4. Autoclave steam sterilization cycles, Part 8: Bowie-Dick \& vacuum leak test cycle. Consolidated Sterilizer Systems website. https://consteril.com/ autoclave-steam-sterilization-cycle-bowie-dick-test/. Accessed March 24, 2020.

5. Verify Bowie-Dick test pack technical data. Steris website. https://ww1.steris. com/onbDocs/V419/1298/688105.pdf. Accessed March 24, 2020.

6. Bowie-Dick simulation test. gke Europe website. https://www.gke.eu/en/ bowie-dick-simulation-test.html Accessed March 25, 2020.

7. Basu D, Bhattacharya S, Mahajan A, Ramanan VR, Chandy M. Sterilization indicators in central sterile supply department: quality assurance and cost implications. Infect Control Hosp Epidemiol 2015;36:484-486.

8. gke batch monitoring system. gke Australia website. https://www.gkeaustralia. com/batch-monitoring. Accessed March 24, 2020.

\title{
Enhanced survival of ST-11 carbapenem-resistant Klebsiella pneumoniae in the intensive care unit
}

\author{
Ying Liu MMed ${ }^{1,2}$ (1), Xiaoxia Zhang $\mathrm{MB}^{1,2}$, Lin Cai BN ${ }^{4}$ and Zhiyong Zong PhD, MBBS ${ }^{1,2,3}$ \\ ${ }^{1}$ Center for Infectious Diseases, West China Hospital, Sichuan University, Chengdu, China, ${ }^{2}$ Center for Pathogen Research, West China Hospital, Sichuan \\ University, Chengdu, China, ${ }^{3}$ Department of Infection Control, West China Hospital, Sichuan University, Chengdu, China and ${ }^{4}$ Intensive Care Unit, West China \\ Hospital, Sichuan University, Chengdu, China
}

To the Editor-Klebsiella pneumoniae, a gram-negative bacterium of the Enterobacteriaceae, is a well-known and major pathogen. Carbapenems are the mainstream agent of choice against K. pneumoniae-producing extended-spectrum $\beta$-lactamases (ESBLs). However, carbapenem-resistant K. pneumoniae (CRKP) has increased rapidly worldwide and has become an urgent threat to public health. CRKP belongs to various sequence types (STs),

Author for correspondence: Zhiyong Zong, E-mail: zongzhiy@scu.edu.cn

Cite this article: Liu Y, et al. (2020). Enhanced survival of ST-11 carbapenem-resistant Klebsiella pneumoniae in the intensive care unit. Infection Control \& Hospital Epidemiology, 41: 740-742, https://doi.org/10.1017/ice.2020.68 and ST-11 is the dominant type of CRKP in China. ${ }^{1}$ However, the factors contributing to the dominance of ST-11 remain largely unknown. Admission to the intensive care unit (ICU) and prolonged length of stay in the ICU are major risk factors for CRKP infection..$^{2-4}$ We hypothesized that ST-11 may have better survival in the ICU than other types of CRKP. We investigated the survival of ST-11 CRKP, a few other types of CRKP, and carbapenemsusceptible K. pneumoniae (CSKP) on a polyvinyl chloride (PVC) surface at the room temperature and humidity in a real ICU situation.

We selected 6 ST11 CRKP strains, 4 CRKP of other STs (ST1, ST15, ST37, and ST45) and 1 CSKP strain (ST1229) for study. Bacteria from frozen stocks were cultured on Luria-Bertani (LB) agar overnight at $37^{\circ} \mathrm{C}$. A single colony of each strain was incubated in $5 \mathrm{~mL} \mathrm{LB}$ broth for $\sim 3-5$ hours to midexponential growth 